\title{
Serum neurofilament light chain is a discriminative biomarker between frontotemporal lobar degeneration and primary psychiatric disorders
}

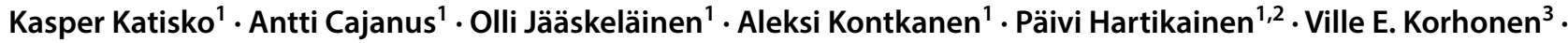 \\ Seppo Helisalmi ${ }^{1}$ - Annakaisa Haapasalo ${ }^{4}$ Heli Koivumaa-Honkanen ${ }^{5,6}$ - Sanna-Kaisa Herukka ${ }^{1,2}$. \\ Anne M. Remes ${ }^{1,2,7,8} \cdot$ Eino Solje $^{1,2}$ (D)
}

Received: 5 September 2019 / Revised: 30 September 2019 / Accepted: 30 September 2019 / Published online: 8 October 2019

(c) The Author(s) 2019

\begin{abstract}
Due to the significant clinical overlap between frontotemporal lobar degeneration (FTLD) spectrum disorders and lateonset primary psychiatric disorders (PPD), diagnostic biomarkers reflecting the different underlying pathophysiologies are urgently needed. Thus far, elevated cerebrospinal fluid (CSF) levels of neurofilament light chain (NfL) have been reported in various neurological conditions. Furthermore, recent advancements in ultrasensitive analytical methods (e.g., single molecule array, Simoa) have enabled sensitive and less invasive NfL detection also from blood samples. In this study, we evaluated the potential of serum NfL (sNfL) as a diagnostic tool between FTLD and PPD. We analyzed sNfL levels with Simoa from 125 participants including patients from FTLD $(n=91)$ and PPD $(n=34)$ spectra. Our results show that sNfL levels are higher in the FTLD group compared to the PPD group as well as in separate clinical subtypes of FTLD compared to different psychiatric manifestations (i.e., mood or psychotic disorders). At single-subject level, discrimination between FTLD and PPD was possible with $80 \%$ sensitivity and $85 \%$ specificity (AUC $=0.850,95 \%$ CI 0.776-0.923), and between behavioral variant frontotemporal dementia (bvFTD) and PPD with $79 \%$ sensitivity and $85 \%$ specificity (AUC $=0.830,95 \%$ CI 0.732-0.908). These findings highlight the potential of sNfL as a discriminating biomarker for FTLD over PPD in patients with wide-ranging behavioral, psychiatric and cognitive symptoms.
\end{abstract}

Keywords Frontotemporal dementia - Frontotemporal lobar degeneration · Psychiatric disorders · Biomarker . Neurofilament light $(\mathrm{NfL}) \cdot \mathrm{sNfL}$

Electronic supplementary material The online version of this article (https://doi.org/10.1007/s00415-019-09567-8) contains supplementary material, which is available to authorized users.

Eino Solje

eino.solje@uef.fi

1 Institute of Clinical Medicine-Neurology, University of Eastern Finland, Yliopistonranta 1C, 1627, 70211 Kuopio, Finland

2 Neuro Center, Neurology, Kuopio University Hospital, Kuopio, Finland

3 Neuro Center, Neurosurgery, Kuopio University Hospital, Kuopio, Finland

\section{Introduction}

The diagnosis of frontotemporal lobar degeneration (FTLD) and especially the behavioral variant frontotemporal dementia (bvFTD) subtype is often challenging, as the heterogeneous clinical manifestation may overlap not only with other

4 A.I. Virtanen Institute for Molecular Sciences, University of Eastern Finland, Kuopio, Finland

5 Institute of Clinical Medicine-Psychiatry, University of Eastern Finland, Kuopio, Finland

6 Department of Psychiatry, Kuopio University Hospital, Kuopio, Finland

7 MRC Oulu, Oulu University Hospital, Oulu, Finland

8 Research Unit of Clinical Neuroscience, Neurology, University of Oulu, Oulu, Finland 
neurodegenerative diseases but also with primary psychiatric disorders (PPD) [1-3]. Thus, biomarkers with a potential to accurately discriminate diseases with wide-ranging neuropsychiatric phenotypes are needed for early and correct diagnosis.

Neurofilament light (NfL), medium (NfM) and heavy $(\mathrm{NfH})$ proteins have recently emerged as potential biomarkers for axonal damage in various neurodegenerative diseases, such as FTLD, Alzheimer`s disease (AD) and Parkinson`s disease (PD) [4]. The diagnostic potential of especially NfL is additionally supported by the fact that current ultrasensitive analytic platforms have enabled NfL detection from peripheral blood samples, providing a convenient and less invasive option for measuring diagnostic markers compared to CSF [5]. A recent study comprising 20 bvFTD patients and 50 psychiatric patients suggested that serum NfL (sNfL) may be used as a biomarker between bvFTD and psychiatric disorders [6].

The aim of this study was to compare sNfL levels in a clinical setting using a larger cohort comprising patients with adult-onset neuropsychiatric or cognitive symptoms, eventually diagnosed either as FTLD spectrum disorder or PPD.

\section{Materials and methods}

\section{Study groups}

All patients (FTLD and PPD) were examined at Kuopio University Hospital between the years 1998-2016 by a neurologist specialized in memory diseases. The PPD patients were diagnosed by a psychiatrist and neurodegenerative disorder was excluded from these patients by a neurologist. Patients (FTLD and PPD) underwent neurological and neuropsychological examination and brain imaging (magnetic resonance imaging (MRI), followed with positron-emission tomography (PET) or single-photon emission computed tomography (SPECT) if necessary), and they were classified according to the current FTLD diagnostic criteria [bvFTD, non-fluent variant primary progressive aphasia (nfvPPA) and semantic variant primary progressive aphasia (svPPA)] [7, 8]. FTLD patients that were diagnosed before 2011 were originally diagnosed according to the Neary 1998 criteria [9] and retrospectively evaluated to meet the Rascovsky or Gorno-Tempini criteria. For bvFTD, only patients fulfilling the clinical diagnosis of at least "probable bvFTD" were included [8] and all the PPA (svPPA and nfvPPA) patients had imaging-supported diagnosis [7]. FTLD-MND patients were diagnosed with probable or definite bvFTD or PPA with concomitant at least clinically possible ALS according to the revised El Escorial criteria [10]. Patients showing signs for other progressive neurodegenerative disease than FTLD or obvious damage to brain (such as stroke) were excluded from this study. The PPD group comprised patients with a severe late onset psychiatric disorder, including psychotic and mood disorders. These patients did not eventually meet at least the probable criteria (11/34 met possible bvFTD criteria but showed no progression and the behavioral disturbance was better accounted for by a psychiatric diagnosis) for any of the FTLD clinical subtypes (at the initial phase or during follow-up). The mean follow-up time for the PPD group was 16 months (median 15 months). Based on these criteria, 125 patients were eventually included in this study. At least the probable criteria for bvFTD were fulfilled in 66 patients, 16 for nfvPPA, four for svPPA, and five for FTLDMND and 34 patients were diagnosed as having a PPD. In the FTLD group, 26 patients had a definite diagnosis due to the C9orf72 repeat expansion (Online Resource Supplementary File 1). Out of the 34 PPD patients, psychotic disorder was present in 18/34 (53\%), mood disorder in 26/34 (76\%) and both mood and psychotic disorder in 10/34 (29\%) of the cases. Specific diagnoses in the psychotic disorder group were: late onset schizophrenia $N=4$, schizoaffective disor$\operatorname{der} N=1$, severe depression with psychotic symptoms $N=6$, bipolar disorder with psychotic depression $N=1$, persistent delusional disorder $N=4$, unspecified non-organic psychosis $N=2$, and in the mood disorder group: severe depression with psychotic symptoms $N=6$, severe depression without psychotic symptoms $N=9$, moderate depression without psychotic symptoms $N=4$, and bipolar disorder $N=7$.

For cognitive and functional evaluation, Mini-Mental State Examination (MMSE) (available from 84 participants of which 55/84 had FTLD) and Alzheimer`s disease co-operative study: activities of daily living inventory (ADCS-ADL) (available from 46 participants, of which $34 / 46$ had FTLD) scores at baseline were tested. Follow-up scores of MMSE were available from 40 patients $(30 / 40$ had FTLD) and of ADCS-ADL from 26 patients (20/26 had FTLD), and a decline rate indicating either cognitive (MMSE) or functional (ADCS-ADL) decline was calculated for these patients (decline in points per months, higher score indicating more rapid decline). The follow-up time between the tests varied between 6 and 33 months (mean 14.5 months) in ADCS-ADL scores and between 3 and 128 months (mean 25.6 months) in MMSE scores.

\section{SNfL analyses (single molecule array)}

SNfL was quantified according to the manufacturer's instructions using the NfL advantage kit for the Quanterix single molecule array (Simoa, Lexington, MA, USA) [11] (Online Resource Supplementary File 1). 


\section{Statistical analyses}

Statistical analyses were performed with IBM SPSS Statistic 25 . The Shapiro-Wilk test and visual inspection were used to evaluate the distribution of the data. Due to non-normal distribution of the $\mathrm{SNfL}$ levels, natural logarithm transformation was used to create normally distributed data (ln-NfL). General linear model, with age as a covariate, was used to compare groups as for the ln-NfL levels. Receiver operating characteristics (ROC) curve analysis was used to calculate area under curve (AUC) with 95\% confidence interval and sNfL cutoff value for optimal sensitivity and specificity. Correlation of $\ln -\mathrm{NfL}$ levels to other variables was analyzed either with Pearson's correlation test (if the other variable was normally distributed) or Spearman's rank correlation test (for non-normally distributed variables). $p$ value $\leq 0.05$ was considered as statistically significant.

\section{Results}

The study population characteristics with mean sNfL levels in each group are described in Table 1. Within the entire cohort (FTLD and PPD combined), sNfL levels showed a positive correlation with age $(r=0.512, p<0.0001)$ and did not differ between genders $(p=0.817)$. Higher sNfL levels correlated with lower MMSE scores at baseline $\left(r_{s}=-0.331, p=0.002\right)$ and also with higher MMSE decline rate $\left(r_{s}=0.521, p=0.001\right)$. There was no correlation between sNfL levels and baseline ADCS-ADL score $\left(r_{s}=-0.044, p=0.773\right)$, but higher sNfl levels correlated with higher ADCS-ADL decline rate $\left(r_{s}=0.509, p=0.009\right)$. Among the FTLD patients, the only significant correlations were the positive correlation of sNfL to age $(r=0.327$, $p=0.002)$ and the negative correlation of sNfL to baseline MMSE score $\left(r_{s}=-0.268, p=0.050\right)$. In the PPD group, there was a significant positive correlation with $\mathrm{SNfL}$ levels and age $(r=0.554, p=0.001)$, and higher sNfL levels correlated with lower ADCS-ADL score at baseline $\left(r_{s}=-0.627\right.$, $p=0.029$ ).

After adjusting for age, the levels of sNfL were higher in the total FTLD group compared to the total PPD group ( $\beta=0.656, p<0.001)$. Additionally, when compared individually, each of the clinical subtypes of FTLD had higher sNfL levels compared to the PPD group: bvFTD vs. PPD ( $\beta=0.538, p<0.001)$, PPA (svPPA and nfvPPA combined) vs. PPD ( $\beta=0.856, p<0.001)$ and FTLD-MND vs. PPD $(\beta=1.389, p=0.001)$. There was no statistical significance in sNfL levels within the PPD group, when comparing patients based on the clinical profile (psychotic vs. mood disorder). The bvFTD group was further compared separately also to PPD subgroups (PPD psychotic and PPD mood), and sNfL levels were significantly higher in bvFTD compared to both of the PPD subgroups: bvFTD vs. PPD psychotic $(\beta=0.425, p=0.014)$ and bvFTD vs. PPD mood ( $\beta=0.632, p<0.001$ ) (Fig. 1). In the ROC analysis between the total FTLD and total PPD groups, the AUC was 0.850 (CI 95\% 0.776-0.923, $p<0.001$ ), and a $19.9 \mathrm{pg} / \mathrm{mL}$ sNfL cutoff level yielded discriminative sensitivity of $80 \%$ and specificity of $85 \%$. When including only bvFTD patients and PPD patients, the values from the ROC analysis were: $\mathrm{AUC}=0.820(\mathrm{CI} 95 \% 0.732-0.908, p<0.001)$ and $19.9 \mathrm{pg} /$ $\mathrm{mL}$ sNfL cutoff level resulted in $79 \%$ sensitivity and $85 \%$ specificity.

Notably, one FTLD patient with bvFTD had a substantially higher sNfL level $(604 \mathrm{pg} / \mathrm{mL})$ compared to the rest of the FTLD group (mean $43.7 \mathrm{pg} / \mathrm{mL}$ ), and this outlier case was excluded from the statistical comparisons. The
Table 1 Study population characteristics and sNfL levels in each group

\begin{tabular}{lrllll}
\hline Group & $N$ & $\begin{array}{l}\text { Age, (years), } \\
\text { mean (SD) }\end{array}$ & Gender, \%F & MMSE, mean (SD) & $\begin{array}{l}\text { sNfL (pg/ } \\
\text { mL), mean } \\
(\mathrm{SD})\end{array}$ \\
\hline FTLD total & 91 & $65.0(8.7)$ & 51.6 & $23.6(4.5)$ & $43.7(36.3)$ \\
BvFTD & 66 & $64.4(9.4)$ & 48.5 & $24.3(3.6)$ & $37.4(32.8)$ \\
PPA & 20 & $67.8(6.4)$ & 60.0 & $21.0(7.1)$ & $54.0(36.4)$ \\
FTLD-MND & 5 & $62.8(4.7)$ & 60.0 & $21.3(3.8)$ & $84.5(49.2)$ \\
PPD total & 34 & $55.7(9.4)$ & 58.8 & $25.4(4.0)$ & $15.5(9.5)$ \\
PPD psychotic & 18 & $57.5(10.7)$ & 72.2 & $24.4(4.2)$ & $18.2(10.8)$ \\
PPD mood & 26 & $54.0(7.7)$ & 50.0 & $25.9(3.9)$ & $13.2(6.3)$ \\
\hline
\end{tabular}

Age is calculated from the date of the blood sample. In the PPD group, ten patients had both PPD psychotic and PPD mood disorder. sNfL was higher in FTLD total compared to PPD total $(p<0.001$, adjusted for age). See Fig. 1 for subgroup comparisons

MMSE Mini-Mental State Examination, $s N f L$ serum neurofilament light, FTLD frontotemporal lobar degeneration, $b v F T D$ behavioral variant frontotemporal dementia, $P P A$ primary progressive aphasia, FTLD-MND frontotemporal lobar degeneration with motor neuron disease, PPD primary psychiatric disorders 

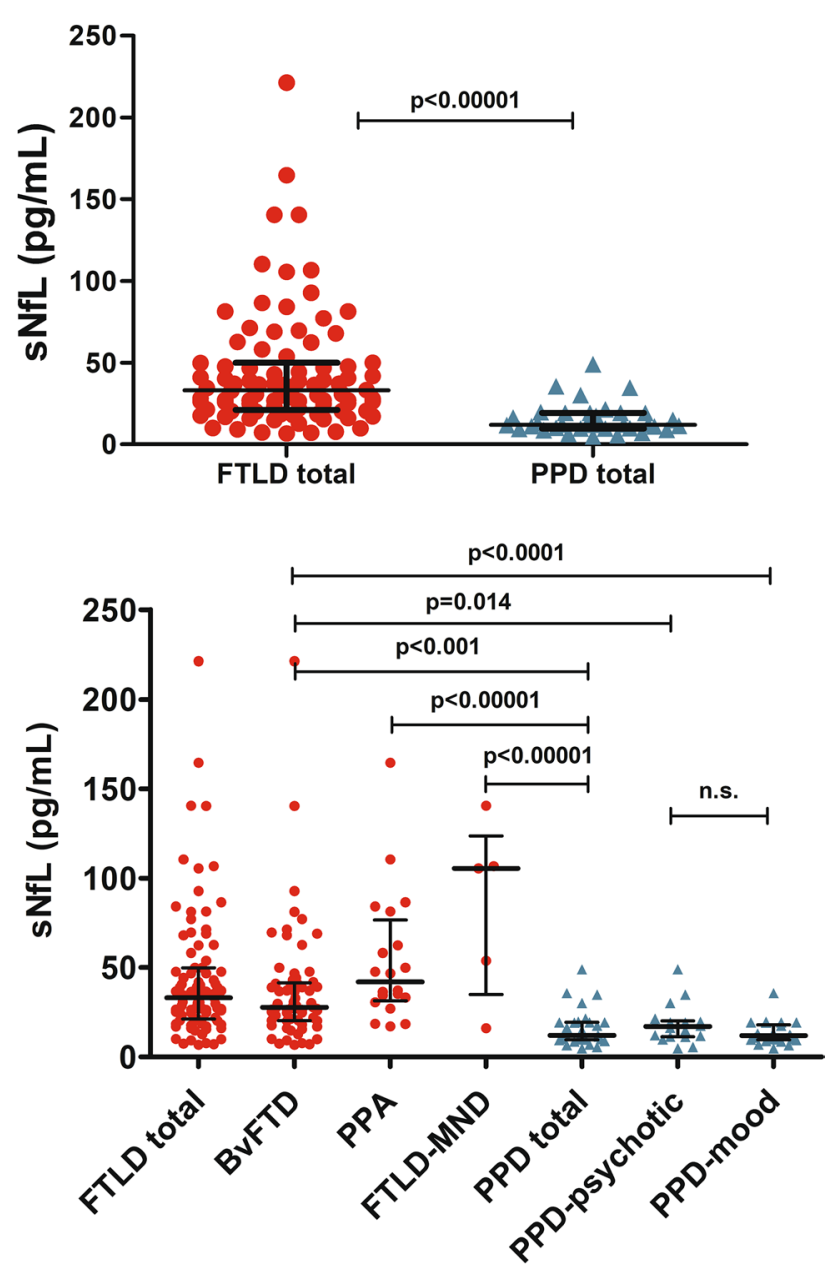

Fig. 1 SNfL concentrations representing each case in each group with group medians and interquartile ranges (IQR). Statistical comparison was performed with general linear model (each of the comparisons made separately) with age as a covariate. $s N f L$ serum neurofilament light, FTLD frontotemporal lobar degeneration, $b v F T D$ behavioral variant frontotemporal dementia, $P P A$ primary progressive aphasia, $F T L D-M N D$ frontotemporal lobar degeneration with motor neuron disease, $P P D$ primary psychiatric disorders

patient was a carrier of the $C 9$ orf 72 repeat expansion, showed wide-ranging behavioral and cognitive dysfunction, and also had psychotic symptoms and a history of prior treatment at a psychiatric ward. The patient did not recall any prior head trauma. Neurological examination showed extrapyramidal symptoms, positive snout reflex, but no signs of motor neuron disease. Also, electroencephalography (EEG) and CSF markers for Alzheimer`s disease or encephalitis were all normal. The patient died within three years after the bvFTD diagnosis. At autopsy, TDP-43 neuropathology without signs of tau or $\beta$-amyloid pathology was confirmed.

\section{Discussion}

To date, this is the largest study reporting sNfL levels in FTLD patients compared to patients with psychiatric disorders. Although recent studies have increasingly reported elevated NfL levels in several neurodegenerative conditions, such as FTLD, AD and PD, the role of NfL in primary psychiatric disorders has remained unclear [4]. The fact that the phenotypes under the FTLD spectrum show a significant clinical overlap with psychiatric disorders [1-3], but substantially differ in the underlying pathophysiology, highlights the importance of minimally invasive pathophysiological biomarkers differentiating these two etiologies. In this present study, we have shown that sNfL levels are higher in FTLD spectrum disorder patients compared to those with PPD, which is in line with a previous report on CSF samples [12] and a recent study with serum samples [6]. As previous studies have already shown a strong correlation between serum and CSF NfL levels in various neurodegenerative disorders [13-15], our study emphasizes the utility of sNfL levels as a potential, minimally invasive differential diagnostic marker between FTLD and PPD.

Our present study revealed that a cutoff level of approximately $20 \mathrm{pg} / \mathrm{mL}$ of sNfL results in a rather high specificity (85\%) in discriminating FTLD and PPD. Almost precisely similar cutoff concentration was suggested in a previous study comparing bvFTD patients and healthy controls [16]. Thus, these findings suggest that PPD may not affect the sNfL cutoff value compared to healthy controls and that the $20 \mathrm{pg} / \mathrm{mL}$ cutoff for sNfL may be used in clinical diagnostics between FTLD and non-neurodegenerative conditions (including PPD). The fact that a few patients in our PPD group showed slightly elevated concentrations (over $20 \mathrm{pg} / \mathrm{mL}$ ) could indicate potential axonal damage in these patients, which might result from the underlying psychiatric disorder, or some other reason, such as minor head trauma. Interestingly, one previous study has shown slightly elevated CSF NfL levels in a subset of patients with bipolar disorder [17], but another study showed that CSF NfL levels in these patients were not associated with clinical outcomes or disease progression [18]. Although the relevance of sNfL in PPD requires further studies, it appears that the possible axonal injury for example in bipolar disorder or psychotic disorders is relatively minor and hence does not significantly affect the potential of using sNfL levels to differentiate between FTLD and PPD. Notably, the positive correlation between NfL levels and age has been observed in healthy controls as well as in patients with a neurodegenerative disease [19-21], including our present study and, therefore, there may be a need for setting age-dependent cutoff values.

Previous studies in FTLD patients have shown that NfL levels also positively correlate with disease severity and 
progression $[22,23]$. In the present study, we found that sNfL levels correlated with the decline rates of MMSE and ADCS-ADL. However, these correlations were observed only in the entire cohort including both FTLD and PPD patients. This could be due to a small sample size, as longitudinal cognitive data were available only for 30 FTLD patients and care should be taken interpreting these nonsignificant results as negative. Moreover, we found that higher sNfL levels were associated with lower MMSE score at baseline in FTLD patients and lower ADCS-ADL score at baseline in PPD patients, indicating that $\mathrm{SNfL}$ is associated with disease severity not only in FTLD but interestingly also in PPD. We also reported a case with an exceptionally high sNfL level. This patient suffered from a rapidly progressive definite bvFTD without motor neuron disease and was first misdiagnosed with PPD. Based on this example, defining the sNfL level may be useful to shorten the diagnostic delay.

The strengths of this study include our novel and clinically relevant cohort setting with a high proportion of definite FTLD cases according to the current diagnostic criteria. Additionally, the heterogeneity in both FTLD and PPD groups in the cohort reflects real-life clinical practice. Compared to a recent paper with a nearly similar study approach (bvFTD vs. PPD) [6], in the present study, we have taken advantage of using a larger bvFTD cohort to confirm the findings related to the potential of sNfL as a discriminative biomarker between bvFTD and PPD. In addition, including also other clinical phenotypes of FTLD provides additional value, as prior psychiatric misdiagnoses are common also in the PPA phenotypes of FTLD [3]. Despite the follow-up for the PPD group, we cannot exclude that some of those patients (especially those with higher sNfL values) might eventually develop FTLD or other neurodegenerative disorder. The limitation of this study is the cohort size, especially considering the limited amount of PPD patients. Additionally, our sNfL data reflect only one cross-sectional time point at the early diagnostic phase and underscore the importance for longitudinal follow-up of the sNfL levels in the future.

In conclusion, our study shows that sNfL levels with a $20 \mathrm{pg} / \mathrm{mL}$ cutoff value are a promising blood-derived discriminating biomarker between FTLD and PPD and that the especially high levels of sNfL in FTLD are associated with a more severe disease. These findings highlight the potential of sNfL as a diagnostic and disease-monitoring tool in FTLD and, most importantly, emphasize the utility of a less invasive serum sample over lumbar puncture for biomarker analyses. Further studies are needed to confirm our findings, especially regarding the relatively low sNfL levels in PPD patients, and to determine the optimal cutoff levels for sNfL as a clinical tool for the discrimination of FTLD from other etiologies.
Acknowledgements Open access funding provided by University of Eastern Finland (UEF) including Kuopio University Hospital. This study was supported by Academy of Finland (Grant Nos. 315459 (Haapasalo), 315460 (Remes)), Yrjö Jahnsson Foundation (Haapasalo, Grant No. 20187070). VTR Grant 5772816 of Kuopio University Hospital (Remes), Finnish Medical Foundation (Katisko), Finnish Brain Foundation (Katisko), Päivikki and Sakari Sohlberg Foundation (Katisko), Maud Kuistila Foundation (Katisko), Finnish Cultural Foundation (Katisko, Cajanus), Olvi Foundation (Cajanus).

\section{Compliance with ethical standards}

Conflicts of interest The authors declare that they have no conflict of interest.

Ethical approval All patients included in this study provided written informed consent prior to enrollment and received genetic counseling before consideration of genetic testing. Research ethics committee of Northern Savo Hospital District approved the study protocol. The study was performed according to the principles of the Declaration of Helsinki.

Open Access This article is distributed under the terms of the Creative Commons Attribution 4.0 International License (http://creativeco mmons.org/licenses/by/4.0/), which permits unrestricted use, distribution, and reproduction in any medium, provided you give appropriate credit to the original author(s) and the source, provide a link to the Creative Commons license, and indicate if changes were made.

\section{References}

1. Galimberti D, Dell'Osso B, Altamura AC, Scarpini E (2015) Psychiatric symptoms in frontotemporal dementia: epidemiology, phenotypes, and differential diagnosis. Biol Psychiatry 78:684692. https://doi.org/10.1016/j.biopsych.2015.03.028

2. Solje E, Aaltokallio H, Koivumaa-Honkanen $\mathrm{H}$ et al (2015) The phenotype of the C9ORF72 expansion carriers according to revised criteria for bvFTD. PLoS ONE. https://doi.org/10.1371/ journal.pone.0131817

3. Woolley JD, Khan BK, Murthy NK et al (2011) The diagnostic challenge of psychiatric symptoms in neurodegenerative disease: rates of and risk factors for prior psychiatric diagnosis in patients with early neurodegenerative disease. J Clin Psychiatry 72:126133. https://doi.org/10.4088/JCP.10m06382oli

4. Khalil M, Teunissen CE, Otto M et al (2018) Neurofilaments as biomarkers in neurological disorders. Nat Rev Neurol 14(10):577589. https://doi.org/10.1038/s41582-018-0058-Z

5. Andreasson U, Blennow K, Zetterberg H (2016) Update on ultrasensitive technologies to facilitate research on blood biomarkers for central nervous system disorders. Alzheimer's Dement Diagn Assess Dis Monit 25(3):98-102. https://doi.org/10.1016/j. dadm.2016.05.005

6. Al Shweiki M, Steinacker P, Oeckl P et al (2019) Neurofilament light chain as a blood biomarker to differentiate psychiatric disorders from behavioural variant frontotemporal dementia. J Psychiatr Res 113:137-140. https://doi.org/10.1016/j.jpsychires 2019.03.019

7. Gorno-Tempini ML, Hillis AE, Weintraub S et al (2011) Classification of primary progressive aphasia and its variants. Neurology 76:1006-1014. https://doi.org/10.1212/WNL.0b013e31821103e6 
8. Rascovsky K, Hodges JR, Knopman D et al (2011) Sensitivity of revised diagnostic criteria for the behavioural variant of frontotemporal dementia. Brain 134:2456-2477. https://doi. org/10.1093/brain/awr179

9. Neary D, Snowden JS, Gustafson L et al (1998) Frontotemporal lobar degeneration: a consensus on clinical diagnostic criteria. Neurology 51:1546-1554. https://doi.org/10.1212/ WNL.51.6.1546

10. Brooks BR, Miller RG, Swash M, Munsat TL (2000) El Escorial revisited: revised criteria for the diagnosis of amyotrophic lateral sclerosis. Amyotroph Lateral Scler 1(5):293-299. https:// doi.org/10.1080/146608200300079536

11. Rissin DM, Kan CW, Campbell TG et al (2010) Single-molecule enzyme-linked immunosorbent assay detects serum proteins at subfemtomolar concentrations. Nat Biotechnol 28:595-599. https ://doi.org/10.1038/nbt.1641

12. Vijverberg EGB, Dols A, Krudop WA et al (2017) Cerebrospinal fluid biomarker examination as a tool to discriminate behavioral variant frontotemporal dementia from primary psychiatric disorders. Alzheimer's Dement Diagn Assess Dis Monit 7:99-106. https://doi.org/10.1016/j.dadm.2017.01.009

13. Kuhle J, Barro C, Andreasson U et al (2016) Comparison of three analytical platforms for quantification of the neurofilament light chain in blood samples: ELISA, electrochemiluminescence immunoassay and Simoa. Clin Chem Lab Med 54(10):1655-1661. https ://doi.org/10.1515/cclm-2015-1195

14. Meeter LH, Dopper EG, Jiskoot LC et al (2016) Neurofilament light chain: a biomarker for genetic frontotemporal dementia. Ann Clin Transl Neurol 3(8):623-636. https://doi.org/10.1002/ acn 3.325

15. Håkansson I, Tisell A, Cassel P et al (2018) Neurofilament levels, disease activity and brain volume during follow-up in multiple sclerosis. J Neuroinflammation 15:209. https://doi.org/10.1186/ s12974-018-1249-7
16. Steinacker P, Anderl-Straub S, Diehl-Schmid J et al (2018) Serum neurofilament light chain in behavioral variant frontotemporal dementia. Neurology 91(15):e1390-e1401. https://doi. org/10.1212/WNL.0000000000006318

17. Jakobsson J, Bjerke M, Ekman CJ et al (2014) Elevated concentrations of neurofilament light chain in the cerebrospinal fluid of bipolar disorder patients. Neuropsychopharmacology 39(10):2349-2356. https://doi.org/10.1038/npp.2014.81

18. Isgren A, Sellgren C, Ekman CJ et al (2017) Markers of neuroinflammation and neuronal injury in bipolar disorder: relation to prospective clinical outcomes. Brain Behav Immun 65:195-201. https://doi.org/10.1016/j.bbi.2017.05.002

19. Mattsson N, Andreasson U, Zetterberg H et al (2017) Association of plasma neurofilament light with neurodegeneration in patients with Alzheimer disease. JAMA Neurol 74(5):557-566. https:// doi.org/10.1001/jamaneurol.2016.6117

20. Sánchez-Valle R, Heslegrave A, Foiani MS et al (2018) Serum neurofilament light levels correlate with severity measures and neurodegeneration markers in autosomal dominant Alzheimer's disease. Alzheimer's Res Ther 10:113. https://doi.org/10.1186/ s13195-018-0439-y

21. Disanto G, Barro C, Benkert P et al (2017) Serum neurofilament light: a biomarker of neuronal damage in multiple sclerosis. Ann Neurol 81(6):857-870. https://doi.org/10.1002/ana.24954

22. Scherling CS, Hall T, Berisha F et al (2014) Cerebrospinal fluid neurofilament concentration reflects disease severity in frontotemporal degeneration. Ann Neurol 75(1):116-126. https://doi. org/10.1002/ana.24052

23. Rohrer JD, Woollacott IOC, Dick KM et al (2016) Serum neurofilament light chain protein is a measure of disease intensity in frontotemporal dementia. Neurology 87(13):1329-1336. https:// doi.org/10.1212/WNL.0000000000003154 